\title{
Static and dynamic heterogeneities in water
}

\author{
By H. Eugene Stanley ${ }^{1}$, Sergey V. BuldyreV ${ }^{1,2}$, \\ Giancarlo Franzese ${ }^{3}$, Nicolas Giovambattista ${ }^{1}$ \\ AND FRANCIS W. STARR \\ ${ }^{1}$ Center for Polymer Studies and Department of Physics, \\ Boston University, Boston, MA 02215, USA (hes@bu.edu) \\ ${ }^{2}$ Department of Physics, Yeshiva University, \\ 500 West 185th Street, New York, NY 10033, USA \\ ${ }^{3}$ Departament de Física Fonamental, Universitat de Barcelona, \\ Diagonal 647, 08028 Barcelona, Spain \\ ${ }^{4}$ Department of Physics, Wesleyan University, Middletown, CT 06459, USA
}

The thermodynamic behaviour of water seems to be related to static heterogeneities. These static heterogeneities are related to the local structure of water molecules and, when properly characterized, may offer an economical explanation of thermodynamic data. 'What matters' most in determining some of the unusual properties of liquid water may be the fact that the local geometry of the liquid molecules is not spherical or oblong, but rather tetrahedral. In respect to static heterogeneities, this local geometry is critical. The dynamic behaviour of water seems to be related to dynamic heterogeneities, which seem to explain the dynamics of supercooled liquid water well.

Keywords: water; static heterogeneities; tetrahedral; dynamic heterogeneities; supercooled liquid water

\section{Puzzling behaviour of liquid water}

Every cook knows that we can superheat water above its boiling temperature. Every scientist knows we can supercool water below its freezing temperature: down to ca. $-40{ }^{\circ} \mathrm{C}$, below which water inevitably crystallizes. In this deeply supercooled region, strange things happen: response functions and transport functions appear as if they might diverge to infinity at a temperature of $c a .-45^{\circ} \mathrm{C}$. These experiments were pioneered by Angell and co-workers over the past 30 years (Debenedetti 2003; Debenedetti \& Stanley 2003; Mishima \& Stanley 1998b; Angell 1983). Down in the glassy region of water (see, for example, figure 1), additional strange things happen, e.g. there is not just one glassy phase (Angell 2004). Rather, just as there is more than one polymorph of crystalline water, so also there appears to be more than one polyamorph of glassy water. The first clear indication of this was a discovery by Mishima et al. (1985): at low pressure there is one form, called low-density amorphous (LDA) ice (Brügeller \& Mayer 1980), while at high pressure there is a new form called

One contribution of 17 to a Discussion Meeting 'Configurational energy landscapes and structural transitions in clusters, fluids and biomolecules'. 


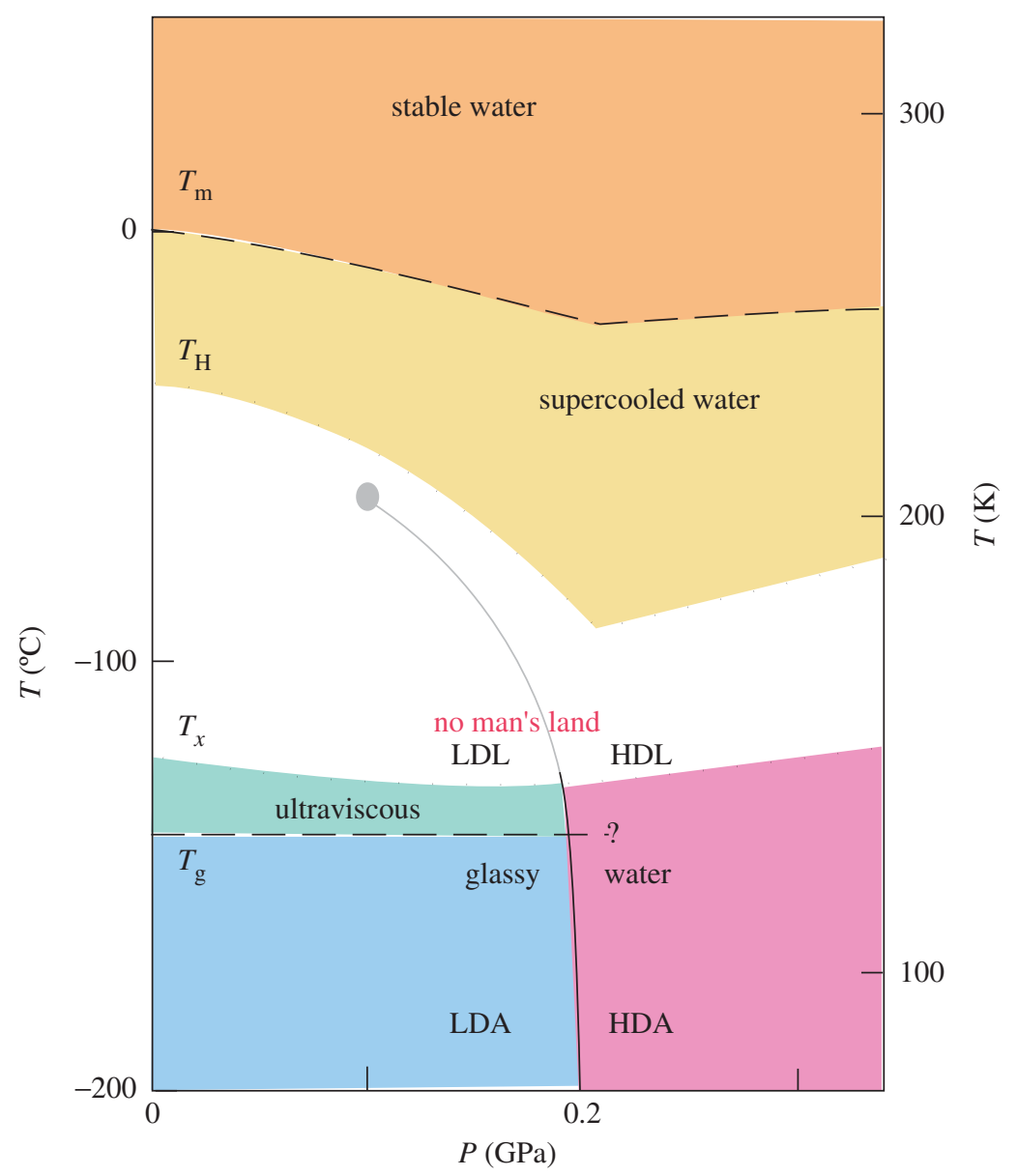

Figure 1. Schematic indicating the various phases of liquid water (colour coded).

(Reproduced courtesy of Dr O. Mishima.)

high-density amorphous (HDA) ice (Mishima et al. 1985). The volume discontinuity separating these two phases is comparable with the volume discontinuity separating low-density and high-density polymorphs of crystalline ice, 25-35\% (Mishima 1994, 1996; Suzuki \& Mishima 2002).

In 1992, Poole and co-workers hypothesized that the first-order transition line separating two glassy states of water does not terminate when it reaches the no man's land, but extends into it (figure 1) (Poole et al. 1992). If experiments could avoid the no man's land connecting the supercooled liquid with the glass, then the LDA-HDA first-order transition line would continue into the liquid phase. This firstorder liquid-liquid (LL) phase-transition line separates two phases of liquid, highdensity liquid (HDL) and low-density liquid (LDL), which are the precise analogues of the two amorphous solids LDA and HDA. Like many first-order transition lines, the LL transition line between non-crystalline phases terminates in a critical point. Above the critical point is an analytic extension of the LL phase-transition line. Called the Widom line, this extension exhibits apparent singularities, i.e. if the system approaches the Widom line, then thermodynamic response functions appear to 
diverge to infinity until the system is extremely close, at which point the functions will round off and ultimately remain finite, as seen in the adiabatic compressibility (Trinh \& Apfel 1980).

\section{Plausibility arguments for static heterogeneities}

That an LL phase transition exists is at least plausible. Plausibility arguments are designed to convince a stranger. My grandmother is not a stranger to me, but she is a stranger to the puzzling behaviour of liquid water. One day she asked, 'Why do liquids condense? Why do molecules floating around in the air suddenly decide to condense?'. To answer, I drew the interaction potential between two water molecules, and explained that there is a minimum at which the water molecules are closer to one another and are more ordered than in the gas, i.e. they have a lower specific volume and a lower entropy. Associated with that minimum is a condensed phase, which we call a liquid.

My grandmother's follow-up question was right on point: 'Why is there only one minimum?'. This was relevant because in liquid water there may very well be two minima. This is because liquid water is a tetrahedral liquid, and two water tetrahedra can approach each other in many different ways. One way is coplanar, as in ordinary hexagonal ice $I_{\mathrm{h}}$, creating a 'static heterogeneity' with a local density not far from that of ordinary ice: $c a .0 .9 \mathrm{~g} \mathrm{~cm}^{-3}$ (figure 2). A second way is altogether different: one of the two tetrahedra is rotated by $90^{\circ}$, resulting in a closer distance where the minimum of potential energy occurs, and hence a static heterogeneity with a local density substantially larger (by $c a .30 \%$ ) than that of ordinary ice (Canpolat et al. 1998). In fact, this rotated configuration occurs in solid crystalline water ('ice VI'), which occurs at very high pressure.

In liquids close to the freezing temperature, there can be heterogeneities with local order resembling that of the nearby crystalline phases. Not surprisingly then, in water at low pressure there are more heterogeneities that have ice-like order (entropy) and density, while at high pressure there are more heterogeneities that have an order and density not altogether unlike ice VI.

The potential that I drew for my grandmother could not represent all the possible relative orientations of two water tetrahedra; rather, it simply had two wells: a deeper, 'high-volume, low-entropy' well corresponding to the LDL and a shallower, 'low-volume, high-entropy' well corresponding to the HDL. Note that the LDL has a higher specific volume and a lower entropy. Therefore, if this double-well potential exists, when water cools, each molecule must decide how to partition itself between these two minima. The specific volume fluctuations increase because of these two possibilities. The entropy fluctuations also increase and the cross-fluctuations of volume and entropy have a negative contribution, i.e. high volume corresponds to low entropy, so that the coefficient of thermal expansion, proportional to these cross fluctuations, can become negative.

The possibility that these static heterogeneities gradually shift their balance between low density and high density as pressure increases is plausible, but need not correspond to a genuine phase transition. There is no inherent reason why these heterogeneities need to 'condense' into a phase, and the first guess might be that they do not condense, an idea which is now called the singularity-free hypothesis (Stanley 1979; Stanley \& Teixeira 1980; Stanley et al. 1981; Sastry et al. 1996).

Phil. Trans. R. Soc. A (2005) 

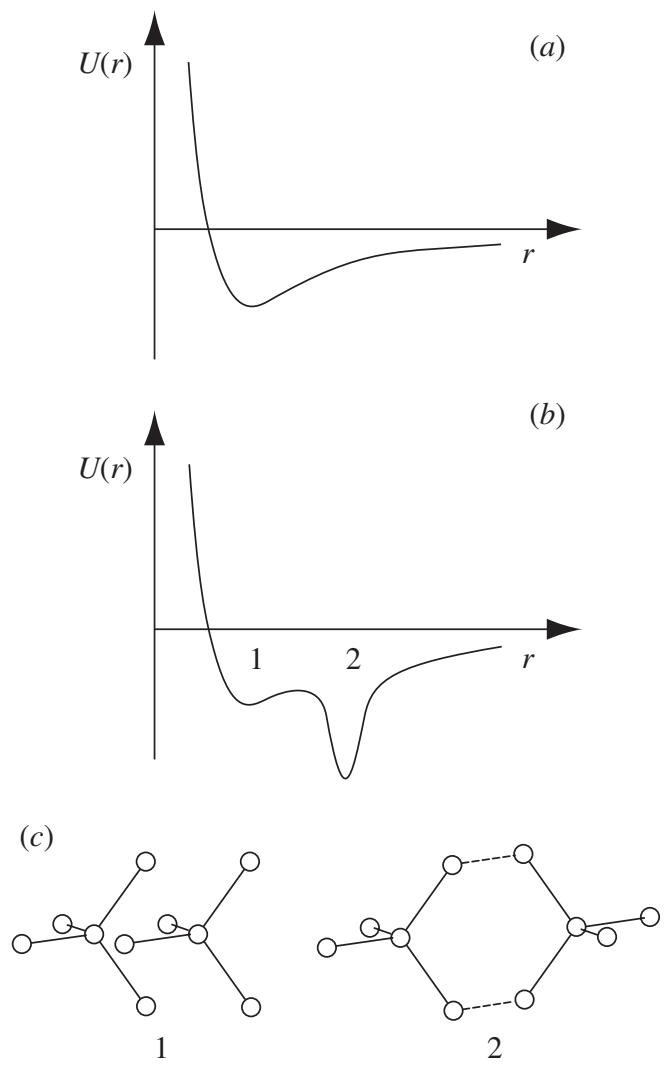

Figure 2. Physical arguments relating to the plausibility of the existence of the known liquid-gas critical point $C$ and the hypothesized LDL-HDL critical point $C^{\prime}$. (a) Idealized system characterized by a pair-interaction potential with a single attractive well. At low enough $T\left(T<T_{\mathrm{c}}\right)$ and high enough $P\left(P>P_{\mathrm{c}}\right)$, the system condenses into the 'liquid' well shown. $(b)$ Idealized system characterized by a pair-interaction potential whose attractive well has two sub-wells, the outer of which is deeper and narrower. For low enough $T\left(T<T_{\mathrm{c}^{\prime}}\right)$ and low enough $P\left(P<P_{\mathrm{c}^{\prime}}\right)$, the one-phase liquid can 'condense' into the narrow outer ' $\mathrm{LDL}$ ' sub-well, thereby giving rise to an LDL phase, and leaving behind the high-density liquid phase occupying predominantly the inner sub-well. (c) Two idealized interaction clusters of water molecules ('Walrafen pentamers') in configurations that may correspond to the two sub-wells of $(b)$.

However, if we suppose that the intramolecular interaction for water molecules has a characteristic energy scale $J_{\sigma}$, at low enough temperature $T$ the ratio $J_{\sigma} / T$ will become large enough to influence the Boltzmann factor sufficiently for the system to condense, giving rise to an LL first-order phase transition line ending in a critical point (figure 3) (Franzese \& Stanley 2002a,b; Franzese et al. 2003). Therefore, the singularity-free scenario is strictly valid only in the limit $J_{\sigma}=0$.

\section{Classic experiments: statics}

The most obvious hallmark of a phase-transition line terminating at a critical point is that there occur fluctuations on all length-scales up to the size of the correlation length. Since the correlation length is substantial even relatively far from the critical 
point, one can see manifestations of critical fluctuations in an entire 'critical region', typically extending by a factor of approximately 2 in all thermodynamic directions away from the critical point.

For example, extremely close to water's gas-liquid critical point, the correlation length is so large that it becomes comparable with the wavelength of visible light, and one can actually see with the naked eye an eerie glow called critical opalescence (Andrews 1869). Since the LL critical point lies well within the no man's land, it is not possible to see critical opalescence in water. Our experimental evidence for this kind of phase transition must therefore be indirect. Nonetheless, the critical region is sufficiently large to significantly perturb measured functions even outside the no man's land. For example, Angell and his collaborators have found linear behaviour when they plot functions double-logarithmically against $T-T_{\mathrm{s}}$, where $T_{\mathrm{s}}=T_{\mathrm{s}}(P)$ is the equation of the Widom Line. Typically, the dimensionless temperature variable $\left(T-T_{\mathrm{s}}\right) / T_{\mathrm{s}}$ does not become smaller than about $7 / 228 \approx 0.03$ (Angell 2004), due to the presence of the line of homogeneous nucleation temperatures $T_{\mathrm{H}}(P)$ which lies just a few degrees above the line of apparent singularity temperatures $T_{\mathrm{s}}(P)$.

When we look at experimental data, we find that they are consistent with the possibility of an LL phase transition. The volume fluctuations are proportional to the compressibility (figure 4), and this compressibility is a spectacularly anomalous function. Below $46^{\circ} \mathrm{C}$, the compressibility starts to increase as the temperature is lowered. This phenomenon is no longer counterintuitive if the double-well potential is correct. Similarly, below $35^{\circ} \mathrm{C}$, the entropy fluctuations that correspond to the specific heat start to increase. Finally, consider the coefficient of thermal expansion, which is proportional to the product of the entropy and volume fluctuations. This is positive in a typical liquid because large entropy and large volume go together, but for water this cross-correlation function has a negative contribution, and as we lower the temperature this contribution gets larger and larger until we reach $4^{\circ} \mathrm{C}$, at which point the coefficient of thermal expansion passes through zero.

\section{Simulation studies on static heterogeneities}

Because the direct experimental study of supercooled water is extremely difficult, several research groups began using computer simulations to look for static heterogeneities. Computer simulations depicting the microscopic nature of local density fluctuations in water were first done in 1982 (Geiger \& Stanley 1982a,b), revealing static heterogeneities whose linear dimension was comparable with the number found experimentally (Bosio et al. 1981) and in simulations (Geiger \& Stanley 1982a). However, these authors did not identify two distinct static heterogeneities. The presence of static heterogeneities with two well-defined local volumes is found in the detailed simulation studies of Errington \& Debenedetti, who report two separate peaks (with an 'isosbestic point') in the histogram of the orientational order parameter (Errington \& Debenedetti 2001). In contrast, for a simple Lennard-Jones fluid they found a single peak.

The hypothesis that the static heterogeneities should condense at a sufficiently low temperature is also supported by simulations using a wide range of molecular potentials, ranging from 'overstructured' potentials such as ST2 to 'understructured' potentials such as SPC/E. Recent work has focused on the newest of all water poten- 


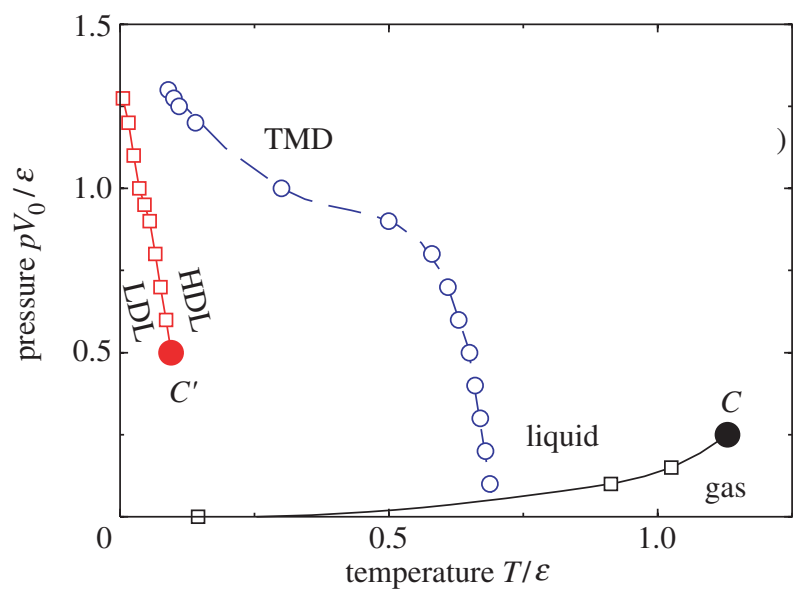

Figure 3. The $P-T$ phase diagram for a water model with intramolecular interaction $J_{\sigma}>0$ calculated in mean field approximation. At high $T$ there is the standard gas-liquid first-order phase transition line (black line) ending in the critical point $C$. At lower $T$ the liquid reaches a maximum in density (dashed blue line marking the temperature of maximum density (TMD)). At lower $T$ there is a first-order phase transition line (red line) between a low-density liquid (LDL) and a high-density liquid (HDL) ending at low pressure in the critical point $C^{\prime}$. Here $J_{\sigma} / \epsilon=0.05, \epsilon$ is the characteristic energy of the van der Waals interaction and $v_{0}$ is the volume per molecule in the gas phase.
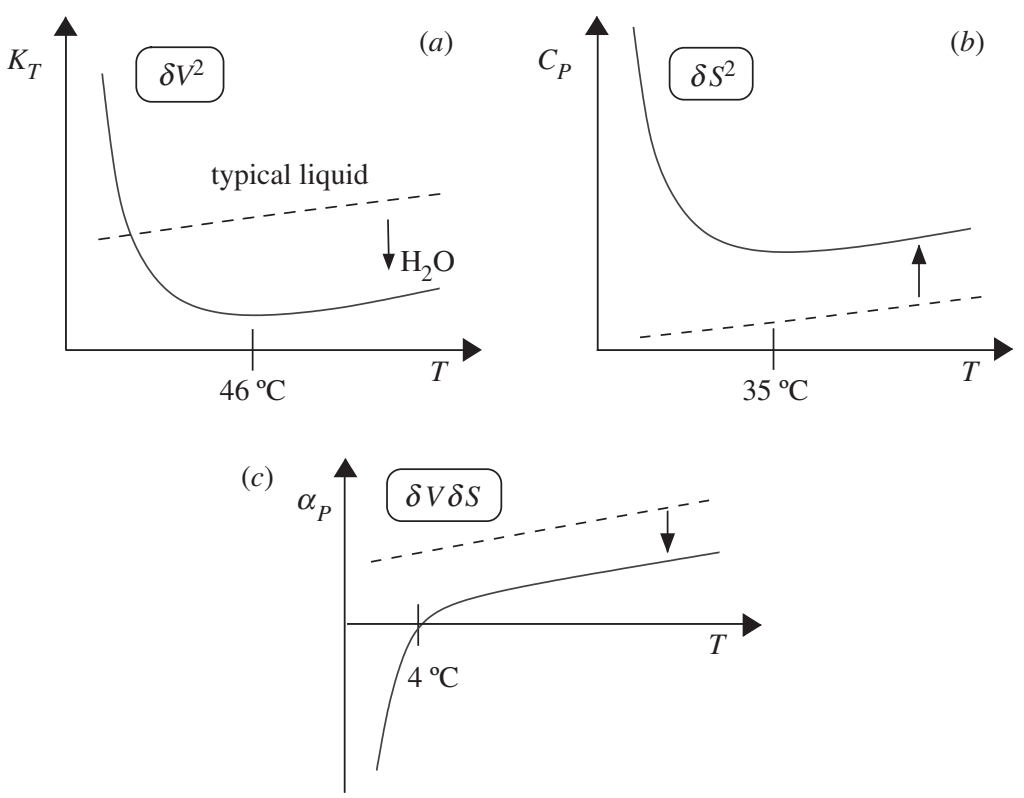

Figure 4. Schematic dependence on temperature of $(a)$ the isothermal compressibility $K_{T},(b)$ the constant-pressure specific heat $C_{P}$ and $(c)$ the thermal expansivity $\alpha_{P}$. The behaviour of a typical liquid is indicated by the dashed line, which, very roughly, is an extrapolation of the high-temperature behaviour of liquid water. Note that, while the anomalies displayed by liquid water are apparent above the melting temperature $T_{\mathrm{m}}$, they become more striking as one supercools below $T_{\mathrm{m}}$. 


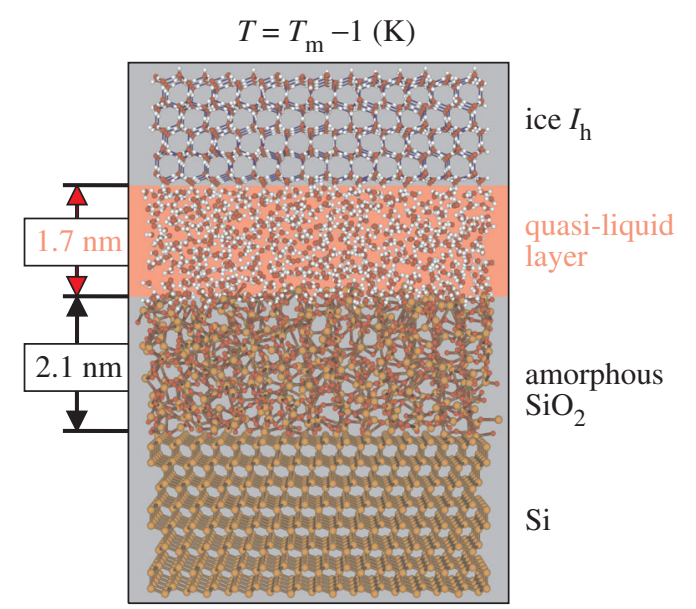

Figure 5. Schematic of the experiments of Reichert and co-workers (Engemann et al. 2004), in which a thin layer of liquid water appears at the interface between ordinary hexagonal ice and an amorphous silica substrate. (Reproduced courtesy of H. Reichert.)

tials, Tip5p (Yamada et al. 2002). Regardless of potential used, all results seem to be consistent with the LL phase-transition hypothesis (Debenedetti 2003).

The LL phase-transition hypothesis does not answer the question 'what matters?', i.e. it does not tell us which liquids should exhibit LL phase transitions and which should not. It has been conjectured that the local tetrahedral geometry of water is what matters, since a tetrahedral local geometry leads to static heterogeneities, which then lead to an LL phase transition (Stanley et al. 2002). But then what about other tetrahedral liquids? If we take silicon, another tetrahedral liquid, Sastry \& Angell (2003) find evidence for an LL phase transition. Similarly, phosphorus (Katayama et al. 2000) and $\mathrm{SiO}_{2}$ (Poole et al. 1997) share properties with liquid water and have a local tetrahedral geometry, and experimental evidence supports the LL phasetransition hypothesis. However, not all liquids with local tetrahedral geometry behave like water (Angell et al. 2000). And, conversely, some liquids which do not possess a locally tetrahedral structure exhibit LL phase transitions (see, for example, the classic works of Aasland \& McMillan (1994) and Brazhkin and collaborators as described, for example, in the Russian articles of Brazhkin et al. (2002)). It has been argued that LL phase transitions are associated with liquids possessing a line in the $T-P$ phase diagram at which the density achieves a maximum (Sciortino et al. 2003; Franzese \& Stanley 2002; Franzese et al. 2001; Skibinsky et al. 2004; Marqués et al. 2003). Nevertheless, the LL phase transition can be observed in model systems without maximum in density, but with two characteristic interaction distances associated to two characteristic interaction energies (Franzese et al. 2001, 2002; Skibinsky et al. 2004).

\section{Recent experiments: statics}

The experimental work of Angell and collaborators shows apparent singularities when experimental data are extrapolated into the no man's land. Mishima and co-workers measured the metastable phase-transition lines of ice polymorphs and found that the slopes of these lines exhibited sharp kinks in the vicinity of the hypothesized 
liquid-liquid phase-transition line as predicted by extrapolation (Mishima \& Stanley 1998a; Mishima 2000; Mishima \& Suzuki 2001). The nature of these kinks can be explained if we take into account the fact that an ice polymorph must melt into a metastable liquid before it can recrystallize into a different polymorph. By the Clausius-Clapeyron relation, the slope of that first-order metastable melting line must be equal to the ratio of the entropy change divided by the volume change of the two phases that coexist. In one phase, the coexistence is always the high-pressure polymorph of ice. The other phase is either HDL or LDL. The volumes and entropies of those two liquids are different, and therefore, as the first-order solid-liquid phasetransition line crosses the hypothesized LL phase-transition line, the slope changes.

The Gibbs potentials of two phases coexisting along a first-order transition line must be equal. We already know the Gibbs potential of all the polymorphs of ice, so we know, experimentally, the Gibbs potential of the LDL and the HDL. From the Gibbs potential of any substance one can obtain, by differentiation, the volume. Thus, if we know the Gibbs potential as a function of temperature and pressure, we know the volume as a function of temperature and pressure, which is called the equation of state. In this way Mishima \& Stanley (1998a) were able to find an experimental equation of state for water deep inside the no man's land. This is, of course, not quite the same as actually measuring the densities of two liquids coexisting at the LL phase-transition line, as was recently observed in phosphorus (Katayama et al. 2000; Monaco et al. 2003), since the Mishima experiments concerned metastable melting lines in which the Gibbs potentials of the two phases are not necessarily equal to each other.

Novel experimental approaches able to probe the behaviour in the previously hardto-probe no man's land may offer the possibility of directly observing distinct phases of liquid water. Very recently, Reichert and collaborators (Engemann et al. 2004) studied at ambient pressure the quasi-liquid layer of a few molecules' thickness that forms spontaneously between ice $I_{\mathrm{h}}$ and a solid amorphous $\mathrm{SiO}_{2}$ substrate even at temperatures moderately below the freezing temperature (figure 5). They designed a new experimental technique at Grenoble by which they were able to measure the density of this quasi-liquid layer, and they found $1.17 \mathrm{~g} \mathrm{~cm}^{-3}$ : a value of the density normally obtained only by placing water under substantial pressure (Soper \& Ricci 2000; Ricci \& Soper 2002). Noting that this value also corresponds to the density of HDA, Engemann et al. (2004) suggested that this high-density liquid may have a structural relationship with HDL. Their work holds promise for new ways to exploit the special properties of confined water to search for experimental realizations of anomalies normally occurring only in the no man's land. In fact, Zanotti et al. (2005) have recently studied, under confinement conditions, water which remains liquid all the way down to the glass-transition temperature, i.e. the no man's land does not exist at all.

It only takes one clear experiment to kill a hypothesis. Recently, Loerting and collaborators claimed to find not two glassy waters, but three (Loerting et al. 2001; Finney et al. 2002). If true, this would seem to destroy the liquid-liquid phasetransition hypothesis because we would no longer have two phases of $\mathrm{H}_{2} \mathrm{O}$.

In the temperature-pressure phase diagram there is a first-order transition line separating two phases of amorphous solid water: low-density (LDA) and high-density (HDA). Experimentally, we can press on the LDA and transform it into HDA, with the volume dropping by $c a .30 \%$. This is reversible: when we relieve the pressure, the 


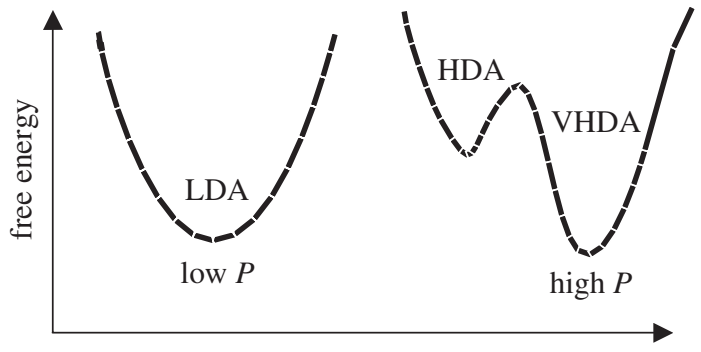

Figure 6. Schematic of the PEL, in which HDA is shown as a valley that is not as deep as, and hence is metastable with respect to, VHDA.

substance returns to the original LDA with hysteresis. This experiment, carried out by Mishima (1994), can be replicated by simulations.

Loerting and collaborators took the same temperature and pressure found in the Mishima experiments and pressed on the LDA to make HDA. They then heated the HDA at constant pressure, from nitrogen temperatures up to $160 \mathrm{~K}$, and cooled it back down. They found that when the HDA was heated from $77 \mathrm{~K}$ to $160 \mathrm{~K}$ the density increased. When it was cooled back down it did not return to the original density, but continued to increase. The structure, as measured by X-rays, was somewhat different. Loerting and collaborators concluded that this was a new form of amorphous solid water, which they called very-high-density amorphous (VHDA) solid water.

Giovambattista et al. (2005) repeated Loerting's experiment on a computer. Loerting and collaborators created HDA at $T=77 \mathrm{~K}$, heated it and cooled it back down. Giovambattista et al. replicated exactly what Loerting and collaborators had found experimentally.

Does this new VHDA form weaken the LL phase-transition hypothesis? Giovambattista et al. have an alternative explanation. They performed a simulation-one that cannot be done in a laboratory. They took the liquid at high pressure and cooled it so rapidly that it jumped across the no man's land. Experimentally, liquid water is extremely difficult to cool rapidly at high pressure (Mishima \& Suzuki 2001) but, with a computer simulation, this extremely rapid cooling can be done. In fact, most computer simulations are of much less than $100 \mathrm{~ns}$ in duration, a time period not sufficient for crystallization to take place (Matsumoto et al. 2002; Yamada et al. 2002). The simulated cooling is continuous and the line extrapolates to the VHDA phase, suggesting that it is the VHDA, not the HDA, that is the stable phase, and that VHDA (not HDA) is the glass connected to HDL by isobaric heating/cooling.

To confirm this interpretation, they measured the structure, i.e. the paircorrelation function $g(r)$ for the two methods of producing VHDA, the first being the method of the Loerting experiment, and the second being the sudden cooling method that can only be employed in simulations. The results were the same.

Thus, we conclude that we do not have three phases of glassy water, but still only two. There is the LDA phase at low pressure; at high pressure there is the highly metastable HDA phase that, when heated, crosses over a barrier and becomes VHDA (figure 6). To confirm this transition, we simply wait. If a glass is highly metastable, it will become something else. If we plot the density as a function of time in nanoseconds, computer simulations show that HDA's density gradually increases until it approaches that of VHDA. 


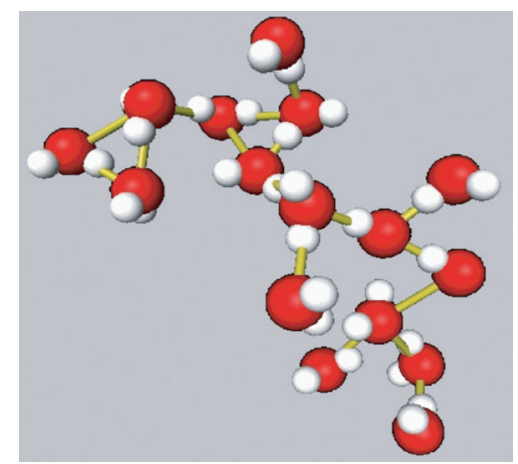

Figure 7. A 'dynamic heterogeneity' (cluster) composed by 16 'mobile molecules' (mobile molecules are defined as $7 \%$ of the molecules which displace most during a time-interval $\Delta t \approx 2 \mathrm{ps}$ ) found in molecular dynamics simulations of supercooled water at $T=260 \mathrm{~K}$. Molecules connected by yellow tubes are neighbour molecules whose oxygen-oxygen distance is less than $0.315 \mathrm{~nm}$, the first minimum in the oxygen-oxygen radial distribution function at $T=260 \mathrm{~K}$. As $T$ decreases, both static and dynamic clusters increase in size, and the precise characterization of these static and dynamic heterogeneities (e.g. their shape, size and lifetime) is an active area of current research.

So the LL phase-transition hypothesis suffered a 'near-death experience'. It appeared that experimentation had detected a new phase of glassy water, when in fact it had detected the stable form of the high-density phase, i.e. VHDA.

\section{Dynamic heterogeneities}

How does water rearrange itself? Glotzer and co-workers (Kob et al. 1997) have shown how simple liquids such as Lennard-Jones liquids restructure themselves in the deeply supercooled region, not 'democratically,' but by a few molecules configured in long, string-like clusters moving cooperatively. This is somewhat like the movement of people in the Tokyo subway. When a hole opens up, someone moves into the hole, leaving a new hole into which someone else moves, and so on. This also happens in water.

The picture of water restructuring involves the mean-squared displacement of molecules as a function of time, in which there is a ballistic regime with water molecules moving like bullets with a constant velocity and a cage-like regime (the Tokyo subway again) where there are no holes and, finally, a diffusive regime. Therefore, macroscopic graphs of mean-squared displacement as a function of time correspond to microscopic properties of those dynamic heterogeneities. Dynamic heterogeneities appear to play a role in the Adam-Gibbs clusters ('cooperatively rearranging regions') that were hypothesized 50 years ago to explain the dynamics of water at low temperature. We seem to have identified those cooperatively rearranging regions (figure 7) and the Adam-Gibbs relations that were hypothesized seem to be borne out (Giovambattista et al. 2003, 2005).

\section{Dynamics and the potential energy landscape}

The dynamics of a system can be related to a potential energy landscape (PEL), the hypersurface obtained by adding one coordinate to phase space, namely the 
potential energy. Seeley \& Keyes (1989) used instantaneous normal-mode analysis to ascertain what controls the dynamics of this potential-energy surface. The way a system point goes from one valley to another valley is what matters in system dynamics. The saddle points or 'mountain passes between valleys' are crucial, so the dynamic properties depend on the number of mountain passes present.

A computer can probe the potential-energy surface and measure dynamic properties such as the diffusion constant. The diffusion constant in liquid water does not depend separately on temperature and on pressure (the two thermodynamic variables) but on a single scaled variable: the fraction of mountain passes (La Nave et al. 2000, 2001; Scala et al. 2000; Starr et al. 2001). For totally different temperatures and pressures (and therefore densities) of liquid water, all the data for this dynamic property collapse on a single curve. This is reminiscent of the data collapse in systems near a critical point. A log-log plot of that same diffusion constant on the $y$-axis as a function of the logarithm of the number of those mountain passes shows that all the density data collapse on a single straight line. Thus there is a power-law dependence on this fraction of mountain passes that characterizes diffusion in liquid water.

When Giovambattista et al. (2002) studied the PEL in great detail at very low temperatures and asked how water rearranges itself, they confirmed some previous findings (Sciortino et al. 1991, 1992), namely, that the hydrogen bond itself is so strong that water would never rearrange itself at these low temperatures. There is not enough energy to break a hydrogen bond. There is, however, enough energy to change a linear hydrogen bond to a bifurcated hydrogen bond. The proton finds itself shared between two oxygens. This rearrangement does not require as much energy as is needed in breaking apart a hydrogen bond.

\section{Discussion}

In summary, in the case of the statics of liquid water, the presence of a local tetrahedral geometry leads to two distinct forms of local order ('static heterogeneities'), differing in specific volume and entropy, with the specific volume and entropy anticorrelated. This fact gives rise to anomalous fluctuations in compressibility, specific heat, and the coefficient of thermal expansion. The possibility that at low enough temperatures these small regions of local order condense into two separate phases (LDA and HDA) is supported by simulations, but is an open question experimentally. Recent experiments suggest there is more than one phase of amorphous solid water, while simulations suggest that one of these phases, HDA, is metastable with respect to another, VHDA, so that in fact there are only two stable phases: LDA and VHDA (Giovambattista et al. 2004a,b, 2005). If in fact there are more than two stable forms of amorphous solid water, then the LL phase-transition hypothesis could be amended to require more than two phases of liquid water-perhaps a less elegant picture, but a possibility consistent with the very recent simulations of Brovchenko et al. (2003) and with the models of Buldyrev \& Stanley (2003) and White (2005).

In the case of the dynamics of liquid water, the actual motion of molecules seems to follow the same pattern as that for simple liquids, with 'dynamic heterogeneities' doing all the action. The potential-energy surface and the number of mountain passes separating valleys in this potential-energy surface seems to be the controlling fac- 
tor. An intriguing open question is the relation between static and dynamic heterogeneities.

So far, the only indirect link is provided by the Adam-Gibbs relation. The static heterogeneities are related to the anomalous increase of water entropy upon compression at constant temperature. Due to the Maxwell relation, the region of this anomaly coincides exactly with the region of the negative thermal expansion coefficient. Interestingly, this region is contained within a wider region of the phase diagram, in which there is an the increase of diffusion coefficient with pressure. The Adam-Gibbs theory relates the diffusion coefficient with configurational entropy and both quantities increase with pressure. The configurational entropy is inversely proportional to the size of dynamic heterogeneities which must thus decrease with pressure. This coincidence suggests that static and dynamic heterogeneities may correlate on the microscopic level as well.

We thank our collaborators, C. A. Angell, M. Canpolat, P. Debenedetti, A. Geiger, P. Kumar, E. La Nave, G. Malescio, M. Mazza, O. Mishima, S. Mossa, P. H. Poole, R. Sadr, S. Sastry, A. Scala, F. Sciortino, R. L. B. Selinger, A. Skibinsky, J. Teixeira and M. Yamada. We also thank the Collaborative Research in Chemistry program of the NSF (CHE-0404673), the Computational Chemistry Program (CHE 0096892), MIUR Cofin 2002 and Firb and INFM Pra GenFdt, Ministerio de Ciecia y Tecnología (Spain) for support, and the Boston University Computation Center for a generous allocation of CPU time. Finally, and very importantly, we thank J. Teixeira for a very critical reading of the manuscript.

\section{References}

Aasland, S. \& McMillan, P. F. 1994 Density-driven liquid-liquid phase separation in the system $\mathrm{Al}_{2} \mathrm{O}_{3}-\mathrm{Y}_{2} \mathrm{O}_{3}$. Nature 369, 633-636.

Andrews, T. 1869 The Bakerian lecture: on the continuity of the gaseous and liquid states of matter. Phil. Trans. R. Soc. A 159, 575-591.

Angell, C. A. 1983 Supercooled water. A. Rev. Phys. Chem. 34, 593-630.

Angell, C. A. 2004 Amorphous water. A. Rev. Phys. Chem. 55, 559-583.

Angell, C. A., Bressel, R. D., Hemmati, M., Sare, E. J. \& Tucker, J. C. 2000 Water and its anomalies in perspective: tetrahedral liquids with and without liquid-liquid phase transitions. Phys. Chem. Chem. Phys. 2, 1559-1566.

Bosio, L., Teixeira, J. \& Stanley, H. E. 1981 Enhanced density fluctuations in supercooled $\mathrm{H}_{2} \mathrm{O}$, $\mathrm{D}_{2} \mathrm{O}$, and ethanol-water solutions: evidence from small-angle X-ray scattering. Phys. Rev. Lett. 46, 597-600.

Brazhkin, V., Buldyrev, S. V., Ryzhov, V. N. \& Stanley, H. E. (eds) 2002 New kinds of phase transitions: transformations in disordered substances. In Proc. NATO Advanced Research Workshop, Volga River. Dordrecht: Kluwer.

Brovchenko, I., Geiger, A. \& Oleinikova, A. 2003 Multiple liquid-liquid transitions in supercooled water. J. Chem. Phys. 118, 9473-9476.

Brügeller, P. \& Mayer, E. 1980 Complete vitrification in pure liquid water and dilute aqueous solutions. Nature 288, 569-571.

Buldyrev, S. V. \& Stanley, H. E. 2003 A system with multiple liquid-liquid critical points. Physica A 330, 124-129.

Canpolat, M., Starr, F. W., Sadr-Lahijany, M. R., Scala, A., Mishima, O., Havlin, S. \& Stanley, H. E. 1998 Local structural heterogeneities in liquid water under pressure. Chem. Phys. Lett. 294, 9-12.

Debenedetti, P. G. 2003 Supercooled and glassy water. J. Phys. Condens. Matter 15, R1669R1726.

Phil. Trans. R. Soc. A (2005) 
Debenedetti, P. G. \& Stanley, H. E. 2003 The physics of supercooled and glassy water. Phys. Today 56, 40-46.

Engemann, S., Reichert, H., Dosch, H., Bilgram, J., Honkimaki, V. \& Snigirev, A. 2004 Interfacial melting of ice in contact with $\mathrm{SiO}_{2}$. Phys. Rev. Lett. 92, 205701.

Errington, J. R. \& Debenedetti, P. G. 2001 Relationship between structural order and the anomalies of liquid water. Nature 409, 318-321.

Errington, J. R., Debenedetti, P. G. \& Torquato, S. 2002 Cooperative origin of low-density domains in liquid water. Phys. Rev. Lett. 89, 215503.

Finney, J. L., Bowron, D. T., Soper, A. K., Loerting, T., Mayer, E. \& Hallbrucker, A. 2002 Structure of a new dense amorphous ice. Phys. Rev. Lett. 89, 503-506.

Franzese, G. \& Stanley, H. E. $2002 a$ Liquid-liquid critical point in a Hamiltonian model for water: analytic solution. J. Phys. Condens. Matter 14, 2201-2209.

Franzese, G. \& Stanley, H. E. $2002 b$ A theory for discriminating the mechanism responsible for the water density anomaly. Physica A 314, 508-513.

Franzese, G., Malescio, G., Skibinsky, A., Buldyrev, S. V. \& Stanley, H. E. 2001 Generic mechanism for generating a liquid-liquid phase transition. Nature 409, 692-695.

Franzese, G., Malescio, G., Skibinsky, A., Buldyrev, S. V. \& Stanley, H. E. 2002 Metastable liquid-liquid phase transition in a single-component system with only one crystal phase and no density anomaly. Phys. Rev. E 66, 051206.

Franzese, G., Marqués, M. I. \& Stanley, H. E. 2003 Intramolecular coupling as a mechanism for a liquid-liquid phase transition. Phys. Rev. E 67, 011103.

Geiger, A. \& Stanley, H. E. $1982 a$ Low-density patches in the hydrogen-bonded network of liquid water: evidence from molecular dynamics computer simulations. Phys. Rev. Lett. 49, 1749-1752.

Geiger, A. \& Stanley, H. E. $1982 b$ Tests of universality of percolation exponents for a 3dimensional continuum system of interacting waterlike particles. Phys. Rev. Lett. 49, 18951898.

Giovambattista, N., Starr, F. W., Sciortino, F., Buldyrev, S. V. \& Stanley, H. E. 2002 Transitions between inherent structures in water. Phys. Rev. E 65, 041502.

Giovambattista, N., Starr, F. W., Buldyrev, S. V. \& Stanley, H. E. 2003 Connection between Adam-Gibbs theory and spatially heterogeneous dynamics. Phys. Rev. Lett. 90, 085506.

Giovambattista, N., Mazza, M. G., Buldyrev, S. V., Starr, F. W. \& Stanley, H. E. $2004 a$ Dynamic heterogeneities in supercooled water. J. Phys. Chem. B 108, 6655-6662.

Giovambattista, N., Angell, C. A., Sciortino, F. \& Stanley, H. E. $2004 b$ Glass-transition temperature of water: a simulation study. Phys. Rev. Lett. 93, 047801.

Giovambattista, N., Stanley, H. E. \& Sciortino, F. 2005 Relation between the very high-density and the high-density phases of amorphous solid water. Phys. Rev. Lett. (In the press.)

Katayama, Y., Mizutani, T., Utsumi, W., Shimomura, O., Yamakata, M. \& Funakoshi, K.-i. 2000 A first-order liquid-liquid phase transition in phosphorus. Nature 403, 170-173.

Kob, W., Donati, C., Plimpton, S. J., Poole, P. H. \& Glotzer, S. C. 1997 Dynamical heterogeneities in a supercooled Lennard-Jones liquid. Phys. Rev. Lett. 79, 2827-2830.

La Nave, E., Scala, A., Starr, F. W., Sciortino, F. \& Stanley, H. E. 2000 Instantaneous normal mode analysis of supercooled water. Phys. Rev. Lett. 84, 4605-4608.

La Nave, E., Scala, A., Starr, Stanley, H. E. \& Sciortino, F. 2001 Dynamics of supercooled water in configuration space. Phys. Rev. E 64, 036102.

Loerting, T., Salzmann, C., Kohl, I., Mayer, E. \& Hallbrucker, A. 2001 A second structural 'state' of high-density amorphous ice at $77 \mathrm{~K}$ and 1 bar. Phys. Chem. Chem. Phys. 3, 5355.

Marqués, M. I., Borreguero, J. M., Stanley, H. E. \& Dokholyan, N. V. 2003 A possible mechanism for cold denaturation of proteins at high pressure. Phys. Rev. Lett. 91, 138103.

Matsumoto, M., Saito, S. \& Ohmine, I. 2002 Molecular dynamics simulation of the ice nucleation and growth process leading to water freezing. Nature 416, 409-413.

Phil. Trans. R. Soc. A (2005) 
Mishima, O. 1994 Reversible first-order transition between two $\mathrm{H}_{2} \mathrm{O}$ amorphs at $-0.2 \mathrm{GPa}$ and 135 K. J. Chem. Phys. 100, 5910-5912.

Mishima, O. 1996 Relationship between melting and amorphization of ice. Nature 384, 546-549.

Mishima, O. 2000 Liquid-liquid critical point in heavy water. Phys. Rev. Lett. 85, 334-336.

Mishima, O. \& Stanley, H. E. 1998a Decompression-induced melting of ice IV and the liquidliquid transition in Water. Nature 392, 164-168.

Mishima, O. \& Stanley, H. E. $1998 b$ The relationship between liquid, supercooled, and glassy water. Nature 396, 329-335.

Mishima, O. \& Suzuki, Y. 2001 Vitrification of emulsified liquid water under pressure. J. Chem. Phys. 115, 4199-4202.

Mishima, O., Calvert, L. D. \& Whalley, E. 1985 An apparently first-order transition between two amorphous phases of ice induced by pressure. Nature 314, 76-78.

Monaco, G., Falconi, S., Crichton, W. A. \& Mezouar, M. 2003 Nature of the first-order phase transition in fluid phosphorus at high temperature and pressure. Phys. Rev. Lett. 90, 255701.

Poole, P. H., Sciortino, F., Essmann, U. \& Stanley, H. E. 1992 Phase behaviour of metastable water. Nature 360, 324-328.

Poole, P. H., Hemmati, M. \& Angell, C. A. 1997 Comparison of thermodynamic properties of simulated liquid silica and water. Phys. Rev. Lett. 79, 2281-2284.

Ricci, M. A. \& Soper, A. K. 2002 Jumping between water polymorphs. Physica A 304, 43-52.

Sastry, S. \& Angell, C. A. 2003 Liquid-liquid phase transition in supercooled silicon. Nature Mater. 2, 739-743.

Sastry, S., Debenedetti, P., Sciortino, F. \& Stanley, H. E. 1996 Singularity-free interpretation of the thermodynamics of supercooled water. Phys. Rev. E 53, 6144-6154.

Scala, A., Starr, F. W., La Nave, E., Sciortino, F. \& Stanley, H. E. 2000 Configurational entropy and diffusivity of supercooled water. Nature 406, 166-169.

Sciortino, F., Geiger, A. \& Stanley, H. E. 1991 Effect of defects on molecular mobility in liquid water. Nature 354, 218.

Sciortino, F., Geiger, A. \& Stanley, H. E. 1992 Network defects and molecular mobility in liquid water. J. Chem. Phys. 96, 3857.

Sciortino, F., La Nave, E. \& Tartaglia, P. 2003 Physics of the liquid-liquid critical point. Phys. Rev. Lett. 91, 155701.

Seeley, G. \& Keyes, T. 1989 Normal-mode analysis of liquid-state dynamics. J. Chem. Phys. 91, $5581-5586$.

Skibinsky, A., Buldyrev, S. V., Franzese, G., Malescio, G. \& Stanley, H. E. 2004 Liquid-liquid phase transitions for soft-core attractive potentials. Phys. Rev. Lett. 69, 061206.

Soper, A. K. \& Ricci, M. A. 2000 Structures of high-density and low-density water. Phys. Rev. Lett. 84, 2881-2884.

Stanley, H. E. 1979 A polychromatic correlated-site percolation problem with possible relevance to the unusual behavior of supercooled $\mathrm{H}_{2} \mathrm{O}$ and $\mathrm{D}_{2} \mathrm{O}$. J. Phys. A 12, L329-L337.

Stanley, H. E. \& Teixeira, J. 1980 Interpretation of the unusual behavior of $\mathrm{H}_{2} \mathrm{O}$ and $\mathrm{D}_{2} \mathrm{O}$ at low temperatures: tests of a percolation model. J. Chem. Phys. 73, 3404-3422.

Stanley, H. E., Teixeira, J., Geiger, A. \& Blumberg, R. L. 1981 Interpretation of the unusual behavior of $\mathrm{H}_{2} \mathrm{O}$ and $\mathrm{D}_{2} \mathrm{O}$ at low temperature: are concepts of percolation relevant to the 'puzzle of liquid water'? Physica A 106, 260-277.

Stanley, H. E., Buldyrev, S. V., Giovambattista, N., La Nave, E., Scala, A., Sciortino, F. \& Starr, F. W. 2002 Statistical physics and liquid water: 'what matters'. Physica A 306, 230-242.

Starr, F. W., Sastry, S., La Nave, E., Scala, A., Stanley, H. E. \& Sciortino, F. 2001 Thermodynamic and structural aspects of the potential energy surface of simulated water. Phys. Rev. E 63, 041201.

Phil. Trans. R. Soc. A (2005) 
Suzuki, Y. \& Mishima, O. 2002 Propagation of the polyamorphic transition of ice and the liquid-liquid critical point. Nature 491, 599-603.

Trinh, E. \& Apfel, R. E. 1980 Sound velocity of supercooled water down to $-33{ }^{\circ} \mathrm{C}$ using acoustic levitation. J. Chem. Phys. 72, 6731-6735.

White, J. A. 2005 Multiple critical points for square-well potential with repulsive shoulder. Physica A, 346, 347-356.

Yamada, M., Mossa, S., Stanley, H. E. \& Sciortino, F. 2002 Interplay between time-temperaturetransformation and the liquid-liquid phase transition in water. Phys. Rev. Lett. 88, 195701.

Zanotti, J.-M., Bellissent-Funel, M.-C. \& Chen, S.-H. 2005 Evidence of liquid-like water between 160 and $300 \mathrm{~K}$. (Submitted). 